Journal of Engineering and Applied Sciences 14 (Special Issue 1): 3927-3930, 2019

ISSN: 1816-949X

(C) Medwell Journals, 2019

\title{
Reliable Secondary Users Selection for Spectrum Treading in Spectrum Secondary Market
}

\author{
${ }^{1}$ Soowook Lee and ${ }^{2}$ Gyanendra Prasad Joshi \\ ${ }^{1}$ Kwangwoon Academy, Kwangwoon University, 20 Kwangwoon-gil, Nowon-gu, 01897 Seoul, Korea \\ ${ }^{2}$ Department of Electrical Engineering and Computer Science, Case School of Engineering, \\ Case Western Reserve University, 10900 Euclid Avenue, Cleveland, OH 44106, USA
}

\begin{abstract}
Selection a reliable spectrum buyer for spectrum treading for cognitive radio networks is a complex decision-making process that requires consideration of a variety of buyer attributes such as price willing to offer, trust and credit score. Although, several algorithms have been utilized for primary user's evaluation and selection, this study proposes a secondary user selection model. The model effectively evaluates secondary users based on the ideal targets set by the incumbent users.
\end{abstract}

Key words: Cognitive radio networks, spectrum sharing, spectrum treading, buyer selection, complex, trust

\section{INTRODUCTION}

Electromagnetic spectrum is a limited natural resource. With the rapid advancement of modern communication system and spectrum licensing system, available spectrum resources are becoming increasingly scarce resource, especially in the Industrial, Scientific and Medical (ISM) frequency bands. Current wireless networks are regulated by fixed spectrum assignment policy. This licensing system is the main reason of the virtual scarcity issue that discourages researchers and venders to develop new technologies in ISM frequency bands. A study about future spectrum usage shows that only mobile traffic (without Machine to Machine (M2M) communication system traffic) is estimated to grow at an annual rate of around $54 \%$ in year 2020-2030. The worldwide traffic volume consumed by M2M services will be $7 \%$ of the total in year 2020 and $12 \%$ of the total in year 2030 (Anonymous, 2015). On the other hand, according to Federal Communication Commission, temporal and geographical variations in the utilization of the assigned spectrum ranges from $15-85 \%$ (Joshi et al., 2009). Current fixed spectrum assignment policy is inefficient and bottleneck for wireless networks development.

The extensive use of wireless communication technology has promoted the development of Cognitive Radio Networks (CRNs) and the dynamic spectrum access mechanism. Moreover, Opportunistic Spectrum Access (OSA) can effectively improve spectrum efficiency and

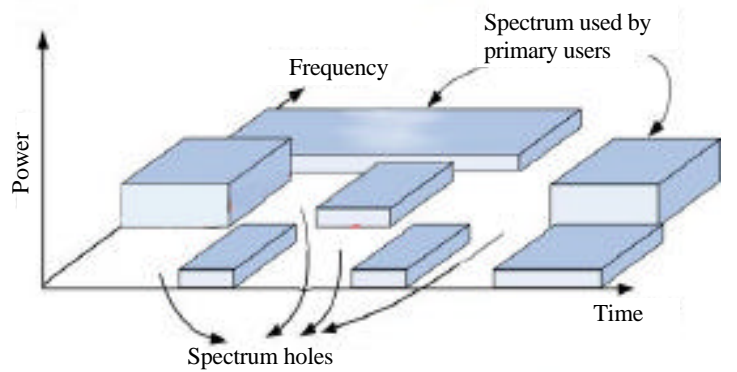

Fig. 1: Spectrum holes and spectrum used by the licensed spectrum users

communication quality (Joshi and Kim, 2016). CRNs and OSA have attracted a great deal of attention recently due to this emerging spectrum scarcity issue.

Cognitive radio networks allow unlicensed users, also called Secondary User (SU), to utilize unutilized or underutilized licensed spectrum bands opportunistically. Here, opportunistically means in a such an intelligent way that do not cause any harmful interference or cause disturbance to the licensed users or the spectrum. The licensed user is also called Primary User (PU) or incumbent users. The incumbents or Primary Users (PU) have the right to use the spectrum anytime, whereas Secondary Users (SU) can utilize the spectrum only when the PU is not using it. The unutilized spectrum band is called spectrum hole or white space.

Figure 1 shows white spaces or spectrum holes and the spectrum used by the incumbent licensed users. In

Corresponding Author: Gyanendra Prasad Joshi, Department of Electrical Engineering and Computer Science, Case School of Engineering, Case Western Reserve University, 10900 Euclid Avenue, Cleveland, OH 44106, USA 


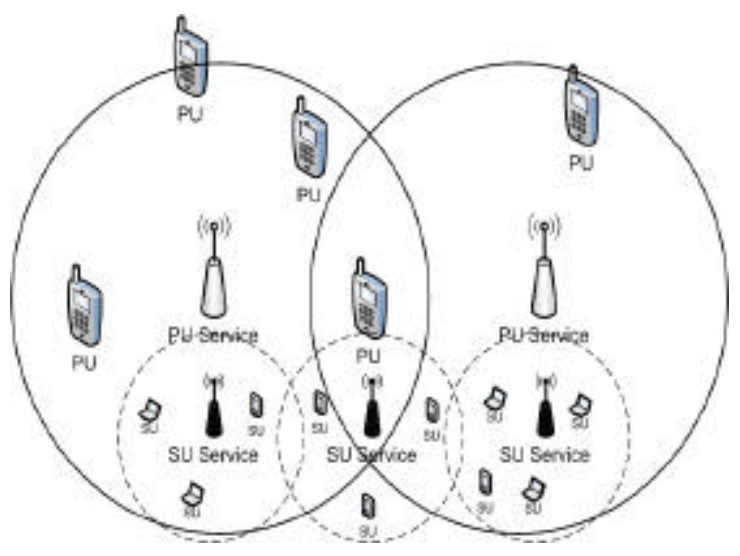

Fig. 2: System structure of primary and secondary users

CRN, SU can use the spectrum band if incumbent licensed user is not using it. However, if licensed user wishes to use the band currently using by $\mathrm{SU}$, the SU have to move to another spectrum hole or stay in the same band altering the transmission power level or using another modulation scheme to avoid the interference to the PU. Figure 2 shows the system structure of primary and secondary users.

Spectrum treading is the process of selling and buying radio resource (e.g., spectrum) in a cognitive radio environment. License holders may charge some reasonable amount to the unlicensed users and allow them to use for the pre-specified duration in specific frequency and location. Unlicensed users can obtain opportunity to use spectrum in very low cost avoiding complicated licensing procedure of the regulatorily body. Spectrum treading approach is a win-win situation for both the license holders and secondary users.

Spectrum treading can be possible directly from the spectrum license holders or from the brokers. A broker can get authority to redistribute spectrum from various licensed users and resales in the secondary market (Zhu et al., 2012).

Except other technical challenges, pricing of the spectrum bands and selection of reliable buyer (or broker) are the major challenges for the licensed users. In this research, we propose a reliable SU selection model based on several parameters. Here, SU means it can be spectrum broker or end user of the spectrum.

Spectrum treading challenges: For the seamless and cooperative communication both parties, the secondary users and primary users require reliable spectrum treading partners. Selection a reliable spectrum buyer for spectrum treading for cognitive radio networks is a complex decision-making process that requires consideration of a variety of attributes such as complementarity, trust, reliability, expertise and price. Selecting reliable partner is a crucial factor of long-term treading.

For spectrum providers, it is a great challenge to find a reliable buyer that can be trusted, willing to pay appropriate price and with good credit score. Similarly, for the buyer it is the other way around, to find the competitive price with the Quality of Service (QoS) is a great challenge. The following factors should be considered for the spectrum treading.

The price determines its revenue for spectrum license holders. Providing spectrum for the second or third parties for opportunistically is costlier in terms of price and QoS. There is always vulnerability of not being available for the license users. There should be some advantages of taking these risks. Also, the maintenance cost incurs to monitor continuously secondary users. Therefore, the price should be at least the amount that can cover these costs. Further, charge of the spectrum provider depends upon number of frequency channels, number of time slots or transmit power. Basically, there are two kinds of costs associated with spectrum treading: Fixed cost is incurred due to the investment in infrastructure and variable cost is incurred due to performance degradation resulting from sharing or selling the spectrum.

In fact, the price paid by SUs depend upon the satisfaction achieved through the usage of the spectrum. The principle of supply and demand reflects the relationship between the demand for spectrum by secondary users and quantity offered by license holders taking into account the price at which the spectrum is sold. In case of multiple sellers, brokers can play vital role to resell the spectrum to the end-users. The sellers want to attract more buyers by providing QoS with competitive price. Among the multiple buyers, everyone wants to buy the best QoS at the lowest possible price. Therefore, it is not easy to select a SU who is willing to offer better price for the offered spectrum because of competition between spectrum sellers. Because anticompetitive practices are unethical and illegal, it is not always possible to fixed the price of spectrum in the free market.

Reliable SU selection model: It is very important for the consistent treading to select a reliable SU or spectrum broker. This research proposes a method to select a SU or 
spectrum broker based on the reputation assessment. Following are the reputation assessment factors selecting SU or spectrum broker:

Credit score (C): Financial strength of the SU, financial background (similar to the Bank's credit scoring). It also may include financial stability of the company for the reasonable period.

Reliability score (R): How reliable the SU or broker is? It can be calculated from the past transaction history with the same company. If this is the first time of the transection then this score is zero. It includes the history of the SU, i.e., how long this $\mathrm{SU}$ is in this business. Is this $\mathrm{SU}$, completed full contract terms in the past? Is the SU has timely payment history?

Recommendation score (a): Recommended by other sources such as other reliable SUs, competitors (or spectrum provider's association, etc., government or authorities, among others. Trust score (.) of the SUs at time $t$ can be calculated as:

$$
\tau(\mathrm{s}, \mathrm{t})=\mathrm{C}(\mathrm{t})+\mathrm{R}(\mathrm{t})+\Re+\mathrm{P} \tau(\mathrm{R}, \mathrm{t}+1)
$$

where, $\mathrm{P}(\mathrm{R}, \mathrm{t}+1)$ is the probability that the $\mathrm{SU}$ will be reliable for the next certain period, can be calculated as:

$$
\mathrm{P}_{\tau}(\mathrm{R}, \mathrm{t}+1)=\frac{(1-\alpha) \sum_{\mathrm{i}=1}^{\mathrm{n}} \tau(\mathrm{s}, \mathrm{t}-\mathrm{n})}{\mathrm{n}}+\alpha \times \tau(\mathrm{s}, \mathrm{t}-1)
$$

Credit score (C) of the SU at time t can be calculated using logistic regression model (Hassan et al., 2015):

$$
C(t)=\frac{e^{B_{0}}+\sum_{i=1}^{n} B_{i} X_{i}}{1+e^{B_{0}}+\sum_{i=1}^{n} B_{i} X_{i}}
$$

where, $B_{1}(0 . i n)$ are the coefficients of the logistic regression model and $\mathrm{X}_{\mathrm{i}}$ are the interval scale variables, such as revenue, loan etc.

SU selection process: Figure 3 shows the selection process of reliable US for spectrum treading. As shown in the block diagram, whenever a primary user receives a request from a secondary user or spectrum broker, it calculates trust score $($.$) as in Eq. 1. If there are multiple$ requests for the same geolocation and time, it compares

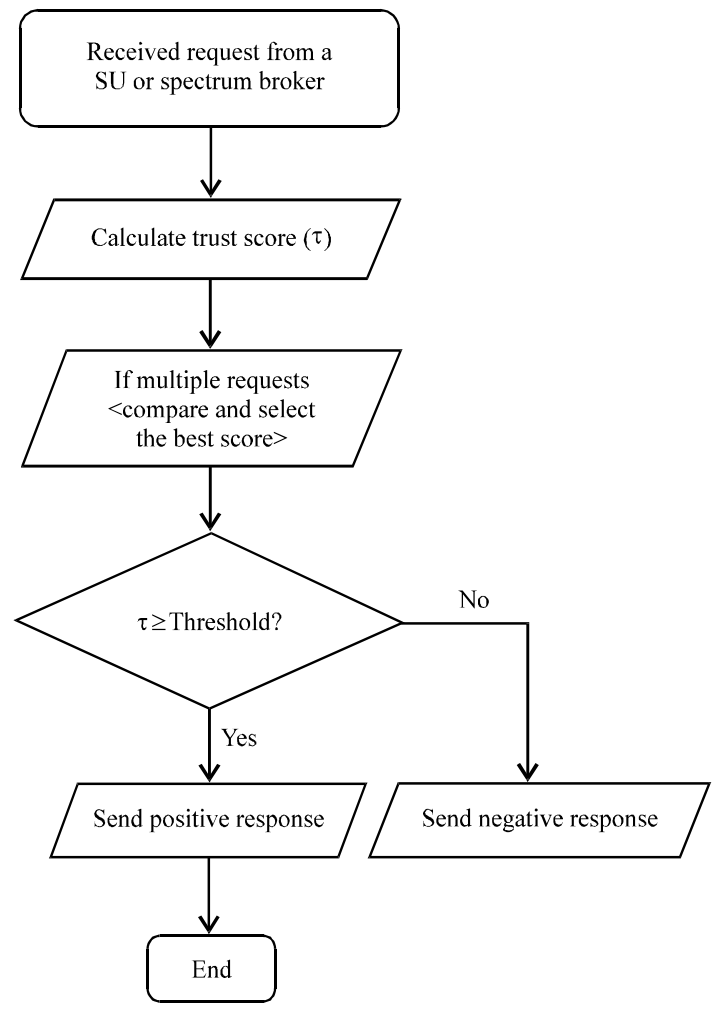

Fig. 3: Selection process of reliable secondary users for spectrum treading.

with those requests and selects the best score. It also compares the trust score with the prespecified threshold value. If the trust score is greater than the prespecified design parameter threshold, it sends positive response to the SU or spectrum broker. Otherwise, it sends negative response to the $\mathrm{SU}$ or spectrum broker.

\section{CONCLUSION}

This research proposes a reliable $\mathrm{SU}$ selection model for spectrum treading. The reliable SU selection model consider buyer's credit score (i.e., financial strength or financial stability), reliability score (i.e., history of the SU, timely payment, completed full contract term (s)), recommendation score (i.e., recommended by other sources such as other reliable SUs, competitors (or Spectrum Provider's Association), government or authorities, etc.) for the selection of SU.

Because there is no such established model for SU selection, therefore, we did not compare our model with any other models. Comparison of our model with some other models is remain for the future research. 


\section{REFERENCES}

Anonymous, 2015. IMT traffic estimates for the years 2020 to 2030. International Telecommunication Union, Geneva, Switzerland. https://www.itu.int/pub/ r-rep-m. 2370

Hassan, R., G. Karmakar, J. Kamruzzaman and B. Srinivasan, 2015. A comprehensive spectrum trading scheme based on market competition, reputation and buyer specific requirements. Comput. Networks, 84: 17-31.

Joshi, G.P. and S.W. Kim, 2016. A survey on node clustering in cognitive radio wireless sensor networks. Sens., Vol. 16, 10.3390/s16091465
Joshi, G.P., S.W. Kim and B.S. Kim, 2009. An efficient MAC protocol for improving the network throughput for cognitive radio networks. Proceedings of the 2009 3rd International Conference on Next Generation Mobile Applications, Services and Technologies, September 15-18, 2009, IEEE, Cardiff, Wales, UK., ISBN:978-0-7695-3786-3, pp: 271-275.

Zhu, K., D. Niyato, P. Wang and Z. Han, 2012. Dynamic spectrum leasing and service selection in spectrum secondary market of cognitive radio networks. IEEE. Trans. Wireless Commun., 11: 1136-1145. 